\title{
Test Results on the Long Models and Full Scale Prototypes of the Second Generation LHC Arc Dipoles
}

\author{
J. Billan, M. Bona, L. Bottura, D. Leroy, O. Pagano, R. Perin, D. Perini, F. Savary, A. Siemko, P. Sievers, G. Spigo, \\ J. Vlogaert, L. Walckiers, C. Wyss \\ CERN- LHC Division, CH-1211, Geneva 23, Switzerland \\ L. Rossi. \\ INFN and University of Milan, LASA Laboratory, via Fratelli Cervi 201, 20090 Segrate (Milano), Italy
}

\begin{abstract}
With the test of the first full scale prototype in JuneJuly 1998, the R\&D on the long superconducting dipoles based on the LHC design of 1993-95 has come to an end. This second generation of long magnets has a $56 \mathrm{~mm}$ coil aperture, is wound with $15 \mathrm{~mm}$ wide cable arranged in a 5 coil block layout. The series includes four $10 \mathrm{~m}$ long model dipoles, whose coil have been wound and collared in industry and the cold mass assembled and cryostated at CERN, as well as one $15 \mathrm{~m}$ long dipole prototype, manufactured totally in industry in the framework of a CERN-INFN collaboration for the LHC. After a brief description of particular features of the design and of the manufacturing, test results are reported and compared with the expectations. One magnet reached the record field for long model dipoles of $9.8 \mathrm{~T}$ but results have not been well reproducible from magnet to magnet. Guidelines for modifications that will appear in the next generation of long magnets, based on a six block coil design, are indicated in the conclusions.
\end{abstract}

\section{INTRODUCTION}

The first series of 10 meter long LHC dipole prototypes ${ }^{1}$, designed and manufactured according to the first LHC conceptual design reported in the so called Pink Book [1], was tested at CERN in the period 1994-95. Those seven long magnets, fully manufactured in industry with relevant variants among them, were all based on $17 \mathrm{~mm}$ wide cables arranged in a six block layout with a $50 \mathrm{~mm}$ coil aperture.

During the construction of those first prototypes and after their test, the dipole design underwent significant modifications, all driven mainly by the goal of saving costs, that are summarized in the two further LHC design studies.

1) White Book [2]: the operational field was fixed at 8.65 $\mathrm{T}$, the magnetic length was increased from 9 to 13 meters, the coil aperture was enlarged to $56 \mathrm{~mm}$, the cable width was reduced to $15 \mathrm{~mm}$ and a five block layout was designed.

2) Yellow book [3]: together with a slight change of the operational field, $8.36 \mathrm{~T}$, and of the magnetic length, $14.2 \mathrm{~m}$, a new coil-collar structure was designed with the conductor

\footnotetext{
Manuscript received September 14, 1998.

1 This series was preceded by a long magnet, named TAP, where the twin apertures concept and the coil-collar-yoke kinematics of the LHC magnet design were tested by using HERA coils at a field considerably lower than foreseen in $\mathrm{LHC}$ at this time.
}

arranged in a new five block layout and an iron shim inserted in the race track shaped twin aluminum collars. Also the distance between the two aperture axes changed from $180 \mathrm{~mm}$ to $194 \mathrm{~mm}$.

A new series of long magnets was then launched in 1995:

- MBL1N1 (N1), 10 m long, Noell, D.

- MBL1N2 (N2), $10 \mathrm{~m}$ long, Noell, D.

- MBL1AJ1 (AJ1), 10 m long, Alsthom- Jeumont, F.

- MBL1AJ2 (AJ2), $10 \mathrm{~m}$ long, Alsthom- Jeumont, F

- MBP1A1 (A1), 15 m long, Ansaldo, I.

The length refers to the cold mass rather than the coils. A cross section of these magnets is shown in Fig. 1 while the main parameters are listed in Table I.

The $10 \mathrm{~m}$ long magnets, called also long models, were intended to be built with minor modifications of the tooling already existing (left from the previous dipole generation) to obtain results from this new design in a two years time. All long models were manufactured in industry up to the collaring of the coils and then completed at CERN (yoking, welding of the shrinking cylinders, cryostating, etc.) in the Magnet Assembly Facility, available from 1996.

The full length prototype had a longer time schedule, and was aimed at testing all the features where the longer length can play a significant role. The prototype, see Fig. 2, was built entirely in industry.

\section{MAGNET DESIGN}

These second generation LHC dipole magnets similarly to the first generation are "twin-apertures", i.e. the two identical coil assemblies are assembled together in the same collars. For these magnets the design variants have been kept to a minimum: for three of them, the insulation was made with all Kapton $^{\mathrm{TM}}$ while for two, N1 and A1, the old Kapton-fiberglass insulation was maintained. A feature of $\mathrm{N} 2$ magnet was the placement of the layer jump of the cable from the inner to the outer layer (ramp) on the same side of the coil where is the splice. The distance between the axes of the $56 \mathrm{~mm}$ in diameter apertures is $194 \mathrm{~mm}$. The stiff race track collars, stiffer than the collars used for the previous generation dipoles, are made out of aluminum alloy. 


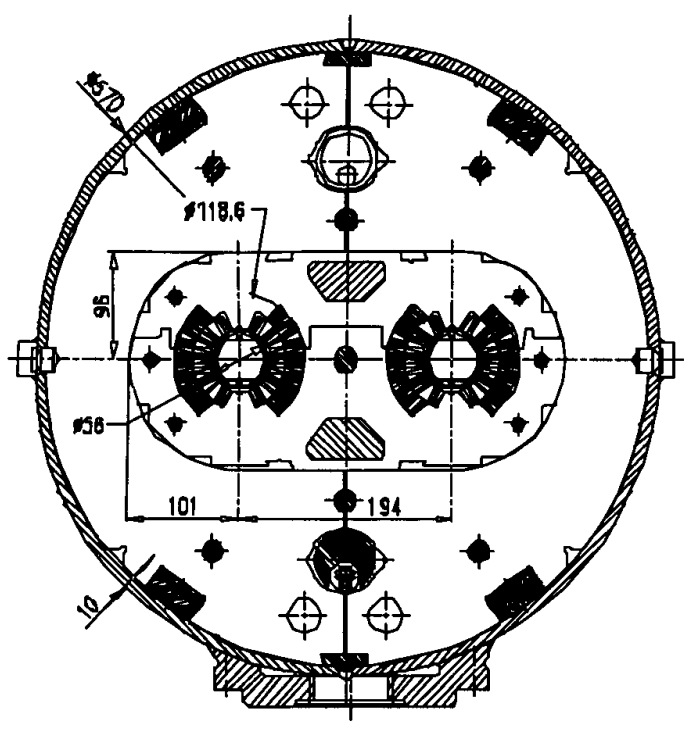

Fig. 1. Cross section of LHC dipoles of the 1995 design ( $2^{\text {nd }}$ generation).

The centering and alignment of the collared coil in the yoke structure is obtained by shaping the inner surface of the yoke so that it closely matches that of collared coil both horizontally and vertically. At room temperature the yoke halves are kept around the collared coil with a force of about $360 \mathrm{kN} / \mathrm{m}$ after the welding of the $10 \mathrm{~mm}$ thick shrinking cylinder made out of $316 \mathrm{LN}$ stainless steel.

The design requires that after welding of the shrinking cylinder the vertical gap between the yoke halves be $0.57 \mathrm{~mm}$ with a narrow tolerance of $\pm 0.05 \mathrm{~mm}$.

The magnet assembly is in principle very robust and permits a good transmission of the horizontal component of the e.m. forces from coil to collar and yoke. The design was meant to obtain a negligible deformation of the collared coil and practically constant field quality throughout excitation.

The NbTi superconductor, operated at $1.9 \mathrm{~K}$, is arranged in two types of cables (one for each layer) both trapezoidally shaped. The width is $15.0 \mathrm{~mm}$, width a compaction factor of $91 \%$. The cables, manufactured by four European industries, were easier to wind than the $17 \mathrm{~mm}$ wide cables of the precedent dipole generation.

TABLE I

MAIN PARAMETERS OF THE LONG MODELS/PROTOTYPE

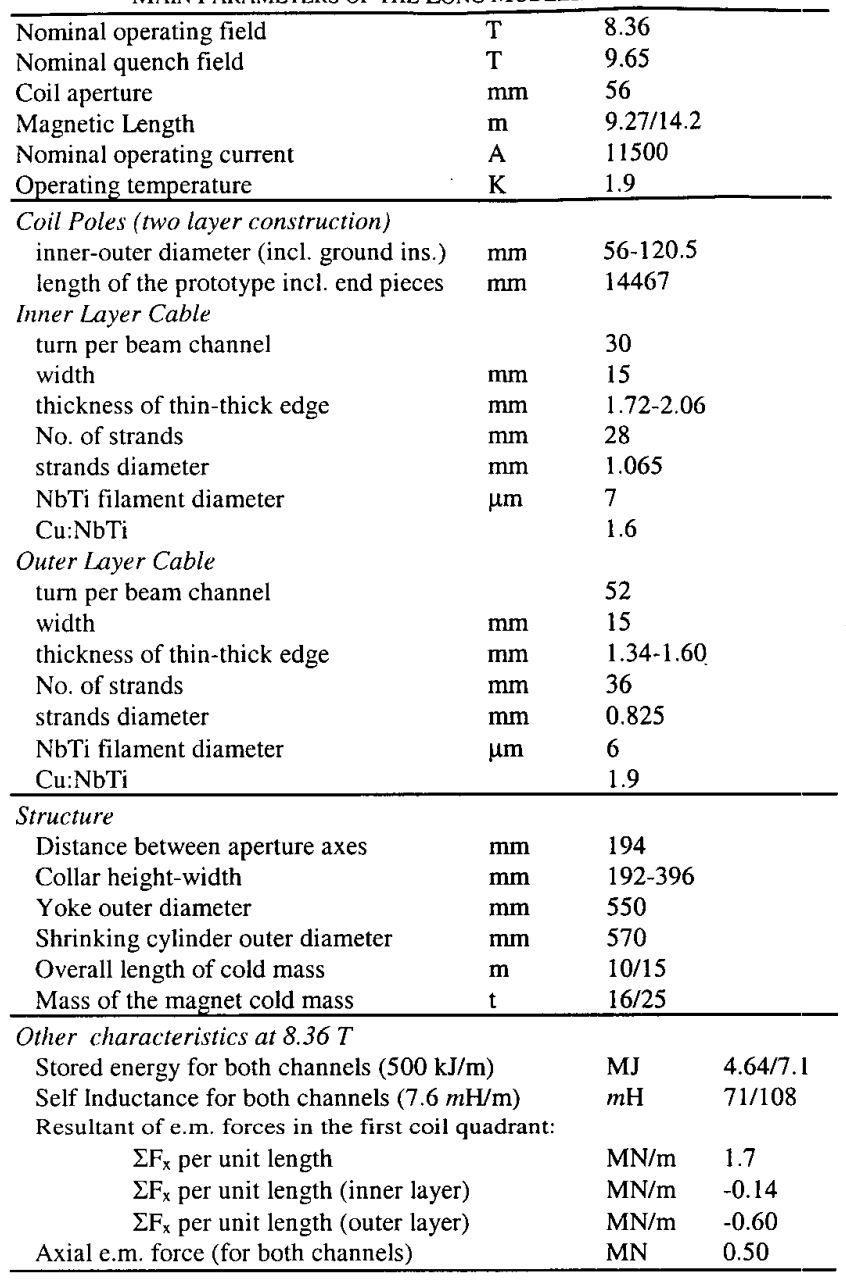

\section{MANUFACTURING FeAtures}

Few manufacturing variants were introduced for evaluation

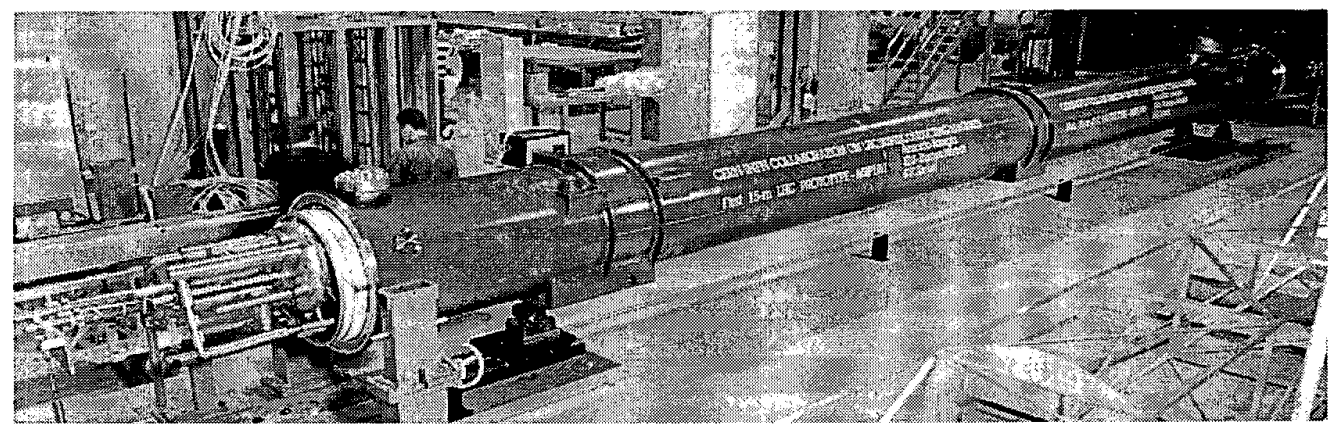

Fig. 2. Photograph of the $15 \mathrm{~m}$ long dipole in its cryostat during preparation for testing. 
in view of the series production [4]. A significant singularity of the $15 \mathrm{~m}$ long prototype is that the coil outer layer is wound directly on the top of the already cured inner layer [5]: In this way the conductor in the splice is kept always under tension. This implies that the cable insulation (a 50\% overlapped 25 $\mu \mathrm{m}$ thick polyimide tapes and a $120 \mu \mathrm{m}$ thick pre-preg glass tape wound around the polyimide insulation with $2 \mathrm{~mm}$ spacing) is submitted twice to the curing cycle in the case of the inner layer (curing temperature of $145^{\circ} \mathrm{C}$ for one hour under $80 \mathrm{MPa}$ of azimuthal compression).

The coil/collar assembly was a little more difficult with respect to the first generation of magnets because in this design the coil is fully contained on the horizontal plane by the very rigid collar, so during the collaring assembly the conductor blocks can move only in vertical direction. For AJ1 and AJ2 magnets collars were assembled on the coil in single female-male pairs, while for the other three magnets they were assembled in packs of 32 pairs.

The collaring was carried out onto a flexible and expandable tube for magnets $\mathrm{N} 1$ and $\mathrm{N} 2$; onto a rigid tube (that had to be removed prior to applying any collaring force) for $\mathrm{A} 1$; without mandrel at all for magnets $\mathrm{AJ} 1$ and $\mathrm{AJ} 2$. After collaring the average azimuthal prestress in the coil was $50 \pm$ $10 \mathrm{MPa}$ for both the inner and the outer layers. During collaring of A1 two heaters were damaged (and one further was shorted during wiring after assembly of the cold mass): this loss, together with an unexpected variation of copper $R R R$ in two outer layers, made the protection scheme of the magnet (see section IV.B) more difficult.

The design value of the yoke gap is $0.57 \mathrm{~mm}$ and during manufacturing of the long dipoles this figure was reached closely with an up-down variation smaller than $0.05 \mathrm{~mm}$ in average but as high as $0.15 \mathrm{~mm}$ as maximum value. For the prototype the average gap was $0.37 \mathrm{~mm}$, with a $0.20 \mathrm{~mm}$ left to right variation, (due to a heavy repair of the welding on one side) that was reduced by means of repeated thermal cycles.

During the assembly phase several problems linked to plastic deformation of the insert were noticed. This resulted in a not easy controllable interference between collared coil and yoke in the zone located above the magnetic insert. The last long model, N2, was assembled with a particular procedure in order to compensate for the above mentioned problems, and to assure collar to yoke contact as foreseen in the design (see values in Table II). $\mathrm{N} 2$ was the dipole with the mechanical structure closer to nominal condition all over the length.

TABLE II

RIGIDITY AND YOKE GAP

\begin{tabular}{llllll}
\hline Name & Rigidity & $\begin{array}{l}\text { Gap } \\
\text { before } \\
\text { welds }\end{array}$ & $\begin{array}{l}\text { Gap } \\
\text { after } \\
\text { welds }\end{array}$ & $\begin{array}{l}\text { Ax. Force } \\
\text { during } \\
\text { welding }\end{array}$ & $\begin{array}{l}\text { Cylinder } \\
\text { Azimut } \\
\text { Stress }\end{array}$ \\
\hline & $\mathrm{GN} / \mathrm{m} \cdot \mathrm{m}$ & $\mathrm{mm}$ & $\mathrm{mm}$ & $\mathrm{MN} / \mathrm{m}$ & $\mathrm{MPa}$ \\
\hline MBL1 N1 & 8.21 & 0.51 & 0.49 & 3.45 & 168 \\
MBL1 N2 & 6.70 & 0.30 & 0.36 & 4.50 & 175 \\
MBL1 JA1 & 9.40 & 0.39 & 0.53 & 5.50 & 165 \\
MBL1 JA2 & 9.87 & 0.54 & 0.61 & 4.40 & 181 \\
MBP1 A1 & not.meas. & $\ldots-\ldots$ & 0.37 & none $^{\dagger}$ & 220 \\
\hline The MBP1 & magnet, was welded with TIG with no vertical force, see text
\end{tabular}

The details of the construction are reported in previous papers [4]-[6]: it is worthwhile to mention that the shrinking cylinders of the four long models were welded at CERN with MAG technique under press, a fast process implemented for the LHC dipoles at the CERN Magnet Assembly Facility, while the prototype (called MBP1A1) was welded in industry with TIG technique without any pressing force.

\section{TEST RESULTS}

\section{A. Field Measurements}

The magnetic field quality was evaluated by measuring the field harmonic components with rotating coils after manufacturing of the collared coil and cold mass and during the test campaign both at room temperature and in superconducting condition. The warm measurements [7] were carried out at a test field of $10 \mathrm{mT}$, while cold measurements [8] were performed over the entire dipole field range.

The correlation between warm and cold measurements is very good, especially for the allowed harmonics $b_{3}$ and $b_{5}$, as well as for the magnetic length, i.e., $\left(\mathrm{L}_{\text {cold }}-\mathrm{L}_{\text {warm }}\right) / \mathrm{L}_{\text {warm }}$ is constant from magnet to magnet. The harmonics measured at $1.9 \mathrm{~K}$ at injection field, averaged along the dipole length, are summarized in Fig. 3. Note that the normal and the skew $2 n$ pole coefficient $b_{n}$ and $a_{n}$ (where $n=1$ is the dipole) are expressed in normalized unit $\left(10^{-4}\right.$ of the main field) at a radius of $10 \mathrm{~mm}$.

The harmonic content of these magnets is not fully satisfactory, even taking into account that field quality was not the main aim of this series. This indicates that probably the tooling for coil construction and for proper positioning of the coil at the beginning of the collaring has to be improved. Some harmonics, while different from company to company, remain constant for different magnets manufactured in the same company, indicating somehow a signature of the tooling used for the assembly. The warm measurements on A1 magnet (the cold measurements have not been carried out, see in the next subsection) show a higher content of harmonics: we think that a very big skew octupole, together with a noticeable skew quadrupole is caused by an asymmetry probably due to difficulty encountered prior and during collaring due to inadequacy of the collaring mandrel.

For all dipoles the field parallelism between the two apertures is very good, better than $0.5 \mathrm{mrad}$, while a global twist, common to the two apertures, of few mrad along the length of the magnet still needs to be improved.

\section{B. Quench results}

The power test campaigns of all dipoles are carried out on the Magnet Test Bench Facility [8] in two different runs separated by a thermal cycle from $1.8 \mathrm{~K}$ to $300 \mathrm{~K}$ and then back to $1.8 \mathrm{~K}$. The magnets are protected against quench induced damages by means of quench heaters and extraction of about $25 \%$ of the energy [9]. The energy extraction actually 


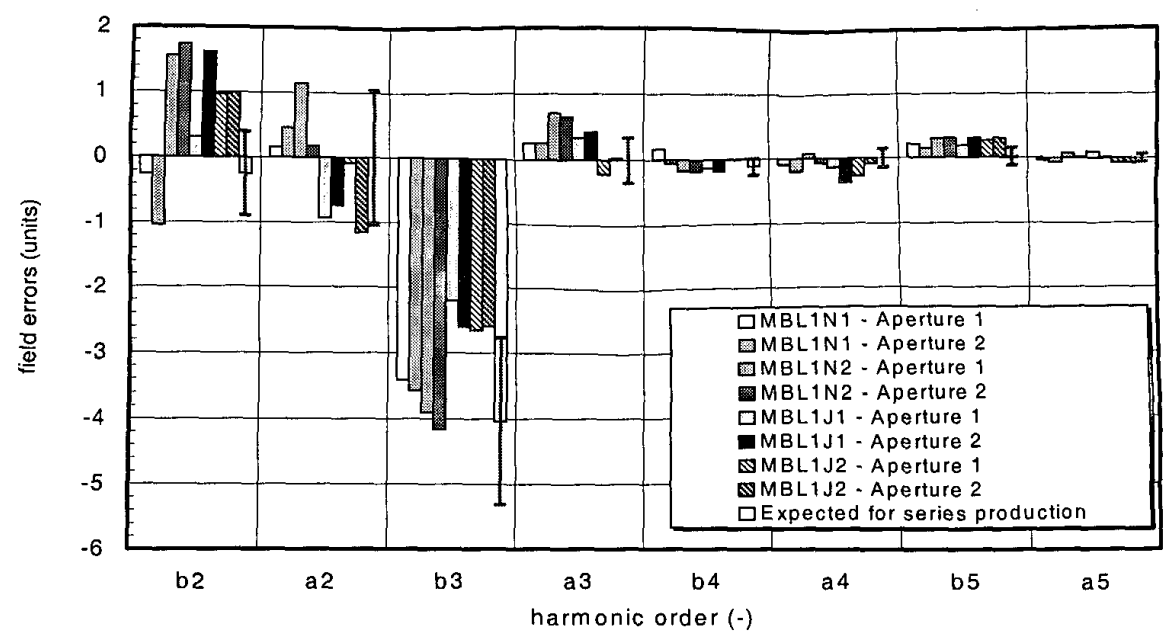

Fig. 3. Values of the average field harmonics at cryogenic temperature for all $10 \mathrm{~m}$ long dipoles measured at $1.9 \mathrm{~K}$

is only about $15 \%$ for the full length prototype, A1. The protection system is triggered when the voltages across the terminals of the poles exceed a voltage threshold of $50 \mathrm{mV}$ for a $10 \mathrm{~ms}$ time interval. For all magnets the quench current was reached with a linear ramp rate of $10 \mathrm{~A} / \mathrm{s}$, except a few quenches on the $15 \mathrm{~m}$ long dipole where the ramp was $2 \mathrm{~A} / \mathrm{s}$ (no significant effect was detected). The training history of each magnet is reported in Fig. 4.

1) Conductor movements and spikes: all magnets have shown spikes in the voltage difference among coil poles and layers. For one of them the amplitude of the spikes is unusually large, more than $1.5 \mathrm{~V}$, while the more quiet magnets have spikes of the order of $100 \mathrm{mV}$. Spikes are detected already at moderate field, above $4 \mathrm{~T}$, and typically they have a maximum around $7 \mathrm{~T}$; at higher field their amplitude tends to decrease. Large spikes are typical of virgin excitation, i.e., they disappear when the ramp is repeated and appear again when the previous maximum current is exceeded. In Fig. 5 the maximum values of the spikes triggering the quench are reported.

This behavior points to conductor displacements resulting in a release of a portion of mechanical energy stored in the windings (bending and shear stresses introduced during collaring). These effects may be enhanced by possible structural weakness, like opening of the yoke gap at high fields (probably observed on N2 magnet).

The two dipoles whose collars have been assembled without mandrel and by single collar pair with a procedure to have the best fitting of the collar to coil, AJ1 and AJ2, have the minimum spikes amplitude indicating how sensitive this design is to the collaring procedure and details.

2) MBLIN1: looking more in detail at the training curve it is significant to note that the N1 magnet started to train at low field levels. After a few quenches it had a sudden increase in the quench level, gaining one tesla in one quench and then approaching very soon the plateau slightly below the short sample limit. This behavior may be due to an initial uncorrect position of a whole coil block that after few quenches was somehow forced in the right position by e.m. forces, see figure on spikes. This hypothesis is partly supported by a difference in the harmonic content measured before first cool down and after reaching high field. The harmonic analysis most probably indicates that the conductor of the inner coil block near the pole was pushed in the correct position by the increasing e.m. forces. The reason of the uncertainty is that the effect of this displacement on $b_{3}$ may be partly compensated by the increase of the inner radius of the coil at midplane under high e.m. forces. This elastic displacement, which makes the coil elliptic (higher deformation modes are under investigation), is of the order of $30-50 \mu \mathrm{m}$ and it generates a harmonic of opposite phase with respect to the outward displacement of the conductor near the pole region. The effect of ellipticity, which was conspicuous enough to almost cancel the increase of $b_{3}$ due to saturation, is mainly due to the lack of radial rigidity of the coil.

Observation of differences between harmonics' before and after the excitation is common for all these magnets, confirming that important conductor adjustments have taken place during the training campaigns.

3) MBL1JA1: the behaviour of the magnet was good in the first training campaign, better than $\mathrm{N} 1$ because AJ1 had no low field quenches. First quench was at $7.9 \mathrm{~T}$ and the following quenches were always well above the nominal field. It is remarkable that the performance reached in the second run, after thermal cycle, is better than expected from short model results: this magnet reached the record field for long model dipole of $9.8 \mathrm{~T}$.

4) MBL1N2: despite the very good accuracy in assernbly, this magnet had a very long training with possible indication of opening of the yoke gap. Its spike behavior was of the type of $\mathrm{N} 1$ but their amplitudes did not settle, see Fig. 5 . 


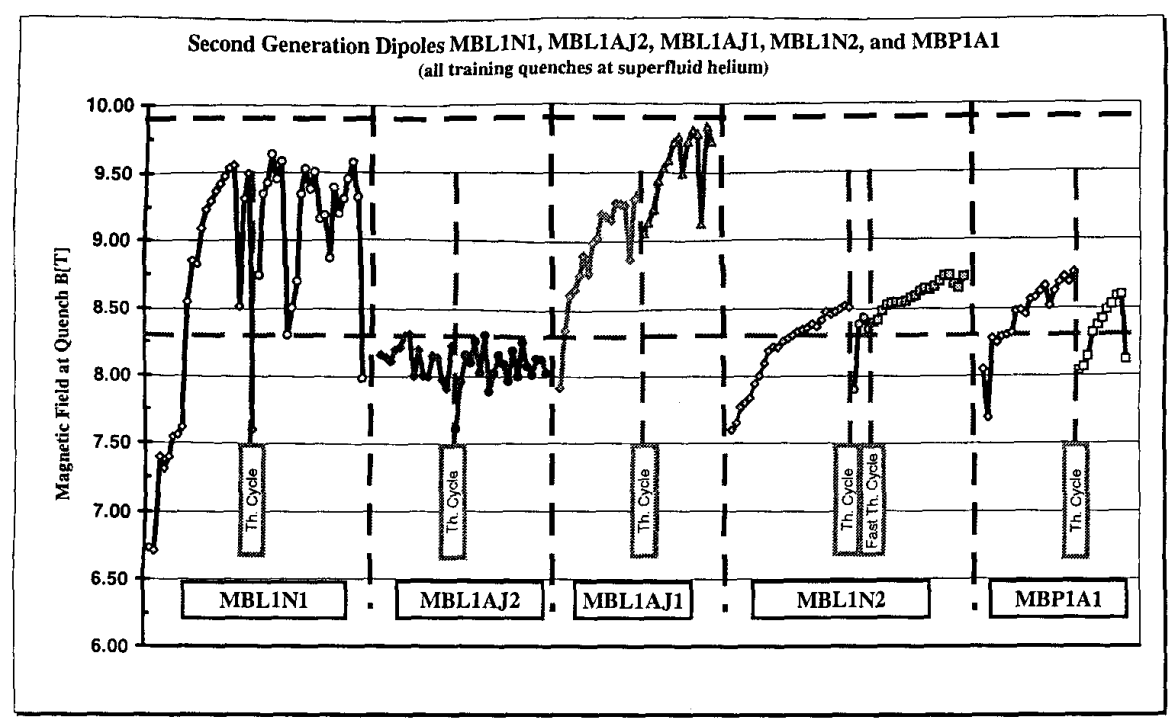

Fig. 4. Training curves for all long dipole magnets for the LHC arcs, built in the period 1995-1997.

5) MBPIAl: This magnet had a good first quench, above 8 $\mathrm{T}$, but suffered from the fact that in the discharge the energy was not released evenly among poles. This happened because of a strong difference in the heaters efficiency, i.e. in the time needed to diffuse the heat from heater to the coil. The time for heat diffusion to coil at high field was found to be about 10$15 \mathrm{~ms}$ shorter for lower poles than for upper poles. This difference in efficiency was fully understood after the second quench, which occurred at $7.7 \mathrm{~T}$, a detraining effect not expected after the first quench at $8.05 \mathrm{~T}$. The detraining was induced by the fact that one of the lower pole, during the first quench, took an energy amount which increased the average temperature by about $20 \mathrm{~K}$ more than in the other poles. This was inferred by two facts: 1) the voltage across the pole that took the largest amount of energy was more than double of the voltages of the other poles; 2 ) the detraining quench initiated exactly in that pole (and not in the pole where quench \# 1 was located). The protection system had to be rearranged before further high field tests. After three quenches the quench protection was brought again to a configuration able to distribute the magnet energy evenly among poles during a quench (the energy amount is $60 \%$ higher than for long models). After this cure the magnet was able to pass the nominal field with a behavior similar to N2. Since after 28 quenches the magnet was damaged, with a coil to ground short circuit, the magnet will be opened to understand the source of this short.

6) MBL1AJ2: the behavior of this magnet was not typical for LHC magnets. The magnet quenched in an irregular way and all quenches after the first five were localized in the outer layer conductor in the proximity of the splice region of a particular superconducting pole, thus indicating that the magnet was limited by a weak splice. This diagnosis was confirmed by a magnet dissection. It is interesting to remark that the first quench of this magnet is the best of this series, over $8.1 \mathrm{~T}$ and this might indicate that, without the weak splice, the magnet behavior would have been good.

7) Detraining: all magnets show detraining, like the short model dipoles when are quenched without energy extraction. The conductor during quench expands and because of the friction, may not come back in its proper position, becoming a weak point to initiate a quench in the following current ramp. This detraining effect is more evident when the training occurs in the field region, above $9 \mathrm{~T}$, as shown in the magnets $\mathrm{N} 1$ and $\mathrm{AJ} 1$. Understanding detraining effects is maybe one of the most important outcomes of the test studies carried out on these long magnets in conjunction with the test on their similar short model dipoles

8) Quench location: there is no predominant quench location for these magnets. The fact that the end and splice regions are not a source of quench as frequent as in the previous generation of long dipoles may indicate that the attention put in the design and manufacturing to these zones has solved this problem. On quench location is to be noted

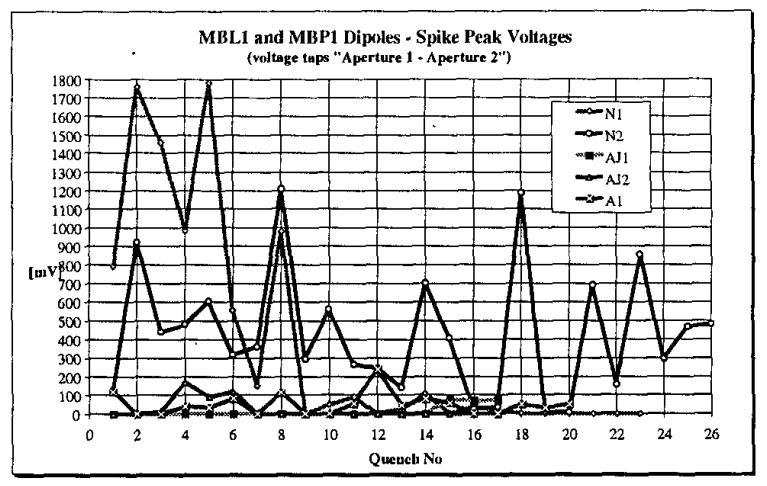

Fig. 5 Amplitudes of the voltage spikes triggering a quench. 
that frequent quenches are located on the outer layers; this is connect to the fact that the hot spot temperature is invariably located in the pole turn of outer layer with relevant thermomechanical effects.

9) Memory: the lack of retraining after a full thermal cycle, is very good for $\mathrm{N} 1$ and exceptionally good for AJ1, acceptable for $\mathrm{N} 2$. It is very poor for A1 that seems to repeat a virgin cycle, behaving as if an important part of coil prestress had been lost.

10) Protection: the magnet protection system in case of quench worked correctly for all five dipoles. For A1 the redundancy of the heater strips allowed rearrangement of the system for its correct functioning. The rearrangement was necessary because of the loss of three heaters before cold test and the non uniform efficiency of remaining strips. The highest hot spot temperature did not exceed the design value of $375 \mathrm{~K}$ in the outer layer conductor. The hot spot temperatures derived from MIITs were within $320-350 \mathrm{~K}$ for quenches at nominal field with full energy deposit. Also the maximum voltages developed across each outer layer during a quench were within the design limit of $500 \mathrm{~V}$.

10) Ramp sensitivity: it is acceptable for all magnets. However it is very different from magnet to magnet, a feature not unexpected since the cables for these magnets, all coated with $\mathrm{SnAg}(5 \%$ wt $\mathrm{Ag})$, were not optimized for this purpose. The ramp sensitivity is low for $\mathrm{N} 1, \mathrm{AJ} 1$ and higher for the other magnets, confirming the necessity of improvement through cable surface oxidation developed at CERN for the next dipole generation.

\section{CONCLUSIONS}

All magnets, apart from AJ2, limited by a weak splice, passed nominal field. The $\mathrm{N} 1$ magnet performed well after a few initial low field quenches and reached $9.65 \mathrm{~T}$, near to its conductor limit and with a good memory effect. The AJI magnet performed very well, reaching $9.8 \mathrm{~T}$ in the second run with an excellent memory. Both magnets reached the plateau with a number of quenches (more than 10 ) in line with the best short ( $1 \mathrm{~m}$ long) model dipole of the same design.

The general conclusion is that the five block coil design is not as robust as expected. All the modifications from its original conception contributed to more difficult conductor positioning (cable less radial and more shear stress between turns) and to more difficult coupling between very rigid collars and coils. It may be that a considerable price was paid for the lack of proper collaring procedures. It is inferred that the structure outside the collared coils probably does not have a primary influence on the performance, at least below $8.5 \mathrm{~T}$. In any case, closing the yoke gap already at room temperature can provide a more reliable structure.

A further consequence of the results of these tests is the need to pass all dipoles through a short power campaign at cryogenic temperatures before installation, to clean up all the sources of quenches below operating field.
The cold tests will probably be necessary even with the expected $0.5 \mathrm{~T}$ improvement in the first quench level of the next generation with six block coil layout[10]. Change of the collaring material from aluminum alloy to stainless steel should reduce the sensitivity to tolerances and improve the repeatability.

The full length dipole will be opened to understand the cause of the short circuit; meanwhile it is used as a unique test bench for experiments on alignment, support system (of the cold mass inside the vacuum chamber) and transportation issues and has already provided precious information.

These dipoles of final aperture and with the curvature, have provided very useful experience for design and manufacturing and their test have constituted the necessary step in understanding the behavior of the high field dipoles at superfluid helium. In view of series production, the acquired experience stresses the importance of tooling of the highest quality, especially in the coil manufacturing and in the collaring process and strictly defined and controlled assembly and quality assurance procedures.

\section{ACKNOWLEDGEMENTS}

The authors acknowledge the continuous support and the very helpful guidance of L. Evans and are very grateful to the CERN teams involved in the construction and testing, as well as the group providing the cryogenic support. A special thanks is due to the teams in industry.

\section{REFERENCES}

[1] The LHC Study Group, "Design Study of the Large Hadron Collider (LHC)" CERN, CERN/91-03, 2 May 1991. Pink Book.

[2] The LHC Study Group, "LHC, the Large Hadron Collider Accelerator Project", CERN, CERN/AC/93/-03(LHC), 8 November 1993. White Book.

[3] The LHC Study Group, "The Large Hadron Collider Conceptual Design", CERN, CERN/AC/95-05(LHC), 20 October 1995. Yellow Book.

[4] L. Bottura et al., "Design, Manufacturing Aspects and Performance of recent $10 \mathrm{~m}$ Long Dipole Superconducting Magnets for the LHC Project", presented at MT-15, October 1997, Beijing, China - LHC Project Report 174, 14 May 1998

[5] M Bona, R. Perin and L. Rossi, "Status of the Construction of the First $15 \mathrm{~m}$ Long Superconducting Dipole Prototype for the LHC", presented at MT-15, October 1997, Beijing, China - LHC Project Report 178, 14 May 1998.

[6] J. Billan et al., "Manufacturing features and performances of long models and first prototype for the LHC projects", EPAC 98, Stockholm, June 1998, in press.

[7] J. Billan et al, " Ambient Temperature Field Measuring System for LHC Superconducting Dipoles", MT-14, 11-16 June 1995, Tampere

[8] J. Billan et al, "Design and Test of the Benches for Magnetic Measurements of the LHC Dipoles", IEEE Trans. on Magn., vol. 30, No.4, p.2658, July 1994

[9] L. Coull et al., "Electromagnetic Behaviour of the LHC Magnet string During a Discharge", EPAC 96, 10-14 June 1996, Sitges, Spain, Vol. 3, p. 2240-2242, 1996A. Siemko et al., "Quench Location in the Superconducting Model Magnets for the LHC by Means of Pick-up Coils", IEEE Trans Appl. Sup., Vol. 5 (2), 1995.

[10]N. Andreev, et al., "State of the Short Dipole Model Program for the LHC", EPAC 98, Stockholm, June 1998, in press. 